\title{
QUERY-SPECIFIC BASED WEB IMAGE RE-RANKING
}

\author{
S.Prince Mary \\ Faculty of Computing Engg, Sathyabama University, Chennai, Tamil Nadu, India \\ princemary26@gmail.com
}

\begin{abstract}
-
This project is based on image search with re-ranking in the web-based by the given query keyword. Normally people used the common search engines for retrieve the image such as google, bing. In that time search engines displayed the large no of images. Users select the particular image from the pool of images. One of the challenges is that the visual features have a lot of similarities and hence do not correlate well. Now a days, people searching the images by image's attributes or reference classes. But different or highly diverse images searching from the web is tuff and poses a lot of inefficiency.

Here project proposed a novel image re-ranking frame work, which the different semantic spaces for different keywords learns automatically. To obtain semantic signatures the featured visuals of images are estimated into their corresponding semantic spaces. In online stage, a comparision of semantic signatures is made and then the images are re-ranked . These semantic signatures are acquired from the semantic space stated by the query keyword. In the postulated query-specific semantic signatures notably enhance the image re-ranking in both accuracy and efficiency.
\end{abstract}

Keywords: Re-ranking, Semantics, Signatures, Web image.

\section{INTRODUCTION}

Information mining is the methodology to investigate the information from diverse condensing and point of view to gather valuable data. Data which can be utilized to expand cuts expenses, income, or both. It is one of scientific instruments to dissect the information. It helps client to dissect information from a wide range of plot, sort it and to distinguish the connections. Essentially, information mining is the methodology to discover examples or relationship among many figures in a huge social databases. Information transforming is a control of info information with an application project to get fancied yield as a graphic, video/audio, numeric or content information documents.

Semantics- Semantics are characterized as a method for communicating the connection between signifiers, in the same way as expressions, words, symbols and signs and their denotation. Linguistic semantics are characterized as a semantics that are utilized to comprehend human representations with the assistance of language. The different sorts of semantics are the semantics of programming dialects, semiotics and formal rationales.
II. SURVEY

[1] Authentication Control for a Semantic Data Repository through an Inference Policy Engine by Abdullah Alamri, Peter Bertok, and James A. Thom In this paper a design of an authorized security model on a semantic model concept This propogate on the individual in the OWL database through a policy engine.Here TBox access control is been made.This paper also provide ABox label based control to evaluate the reasoning and modularization.The future work include the inheritance relations of a role hierarchy and to design a better model to handle detection and conflicting rules.

[2] Improving large-scale search engines with semantic annotations Damaris Fuentes-Lorenzo , Norberto Fernández, Jesús A. Fisteus, Luis Sánchez The search engine lacks efficiency in satisfying ambiguous or synonym queries. In this paper an algorithm is proposed with the help of collaborative semantic annotations and Wikipedia to improve the quality of web search engines to rank the returned results. This paper uses the following methods 1) Resource collaborative tagging 2) Accumulators for low-time response: 3) Query disambiguation. The future work include situations in which new characteristics can be added like the 
possibility of adding new resources directly to the repository, avoiding the second step of manual disambiguation.

[3] Real Time Google and Live Image Search Reranking Jingyu Cui,Fang Wen,Xiaoou Tang. Web-scale image search engines function on purely the surrounding query text which will lead to ambiguous results. This paper uses adaptive visual similarity to re-rank the textbase search results.In this the query image categorization is done and then specific similarity measure is used for combining the image features for re ranking. The future work include combining our work with photo quality re-ranking method.

\section{EXISTING SYSTEM}

The previous system used the common searching methods like enter the query keyword and retrieve the no of images or given the reference class and search different no of images. Web-scale image search engines mainly use keywords as queries and depend on surrounding text for searching images. They agonize from the complexity of query keywords, because it is difficult for users to exactly describe the visual content of target images which use only keywords.

Their search intention will be described by users with some reference images and set of attributes which are already defined. These attributes and concepts are trained offline and which can tolerate with the variation of visual content. Basically, these approaches are only applicable to closed sets of images which are relatively small size, and are not suitable for web-scale image reranking.

All the previous system used the common searching methods like enter the query keyword and retrieve the no of images or given the reference class and search different no of images. Web-scale image search engines mainly use keywords as queries and depend on surrounding text for searching images. They agonize from the complexity of query keywords, because it is difficult for users to exactly describe the visual content of target images which use only keywords.

\section{PROPOSED SYSTEM}

In this paper, a good framework is proposed to rerank the images on web. Insubstitue of defining a universal concept dictionary manually, it learns unique semantic spaces for unique query keywords automatically and individually. The images related to semantic space to be re-ranked can be notably restricted down by the query keyword which will be provided by the user. The query-specific semantic spaces can more precises the model images to be re-ranked, as they have eradicated other potentially countless number of unrelated concepts, which serve only as noise and degrade the re ranking performance on both computational cost and accuracy.

\section{A. Methodology}

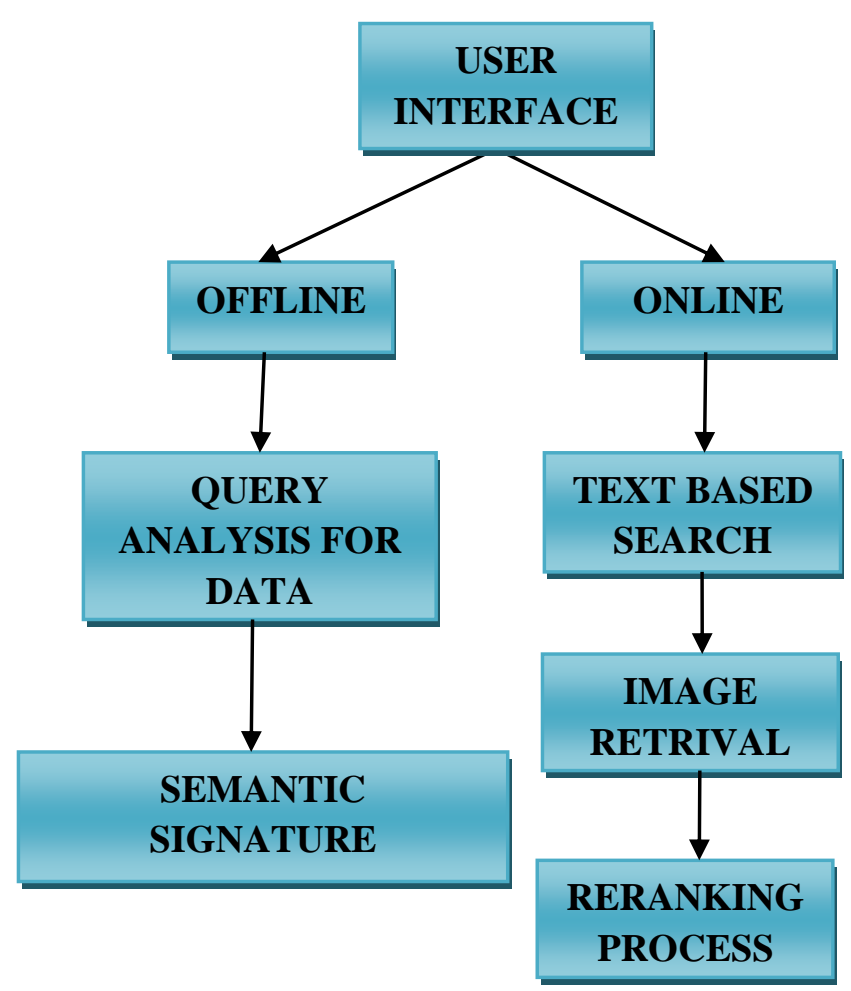

Fig. 1. Methodology

\section{B. User Interface}

WEB-SCALE picture web search tools majorly utilize catchphrases as questions and rely on upon encompassing content to pursuit pictures. They anguish from the many-sided quality of inquiry decisive words, in light of the fact that it is troublesome for clients to precisely depict the visual substance of target pictures which utilize just catchphrases. User connect the query to the API for the retrieve the particular images. Its helps to analyze the query for image retrieval. When a user inputs a textual query and starts to browse the result for text based search, he or she has a intention, to search image 
which could be a exact image or images in a particular category.

\section{Query Analysis For Data Storage}

For each one inquiry decisive word, its reference classes structures the premise of its semantic space. A multi-class classifier on text based and the preparation sets are being prepared from the visual peculiarities for its reference classes and put away logged off. Under an inquiry catchphrase, the semantic signature of a picture is removed by figuring the matching between the reference classes and picture of the question magic word utilizing the prepared multiclass classifier. In the event that there are Various leveled sorts of text based/visual gimmicks, for example, composition, shading and shape, one could acclimatize them together to prepare an one classifier, which removes just single semantic signature for a particular picture. It is likewise teaches an alternate classifier for each one kind of qualities. At that point, the Hierachical classifiers taking into account distinctive sorts of gimmicks concentrate Various leveled semantic marks, which are consolidated at the later phase of picture matching.

\section{Method-Hierarchical Clustering}

\section{Semantic Signature Creation}

The Fig.2 Shows the demonstrate that the recent method can build the re-positioning exactness at the expense of capacity and online closeness effectiveness due to the increment in the span of the semantic marks. Taking into account the statement picture file record, a picture may be connected with more than one inquiry pivotal words, which has diverse semantic spaces. Subsequently, it may have diverse semantic marks. The client chooses which semantic signature to pick for the given information question essential word. Be that as it may, under exceptional inquiry catchphrases, distinctive semantic spaces are utilized. A picture could be acquired in diverse semantic spaces utilizing a few semantic marks. They all need to be put away logged off and registered. Question particular visual semantic space utilizing figuring the semantic mark.

Method: Random walk (RWalk)

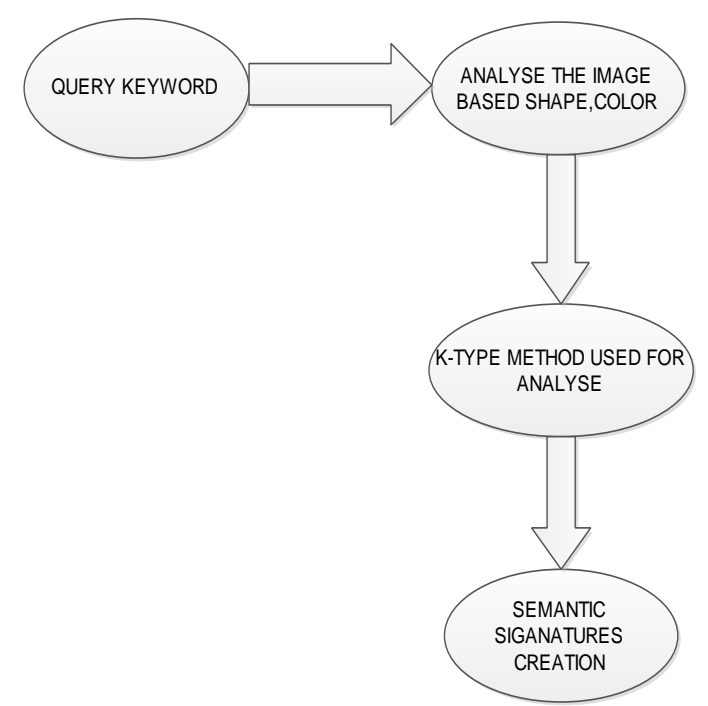

Fig.No.2 SEMANTIC SIGNATURES CREATION

\section{E. Text-based Image search}

Image search is a specialized data search used to find images. To search images, a user mit provide query terms such as image file/link, click on some image or keywords, and the system will send back images which matches the query. To examine the criteria could be color distribution in images, region/shape attributes, meta tags etc. At the online stage, a group of images are obtained by the search engine based on the query keyword.

\section{F. Mage Retrieval}

The reference classes (which speak to diverse ideas) identified with question essential words are consequently found and their preparation pictures are naturally gathered in a few steps of related classes which are similar to specific keywords which were automatically discovered and all their training images are collected automatically in several steps. In Fig.No.3 User enter the keyword to the search engine and get the expansions of the keyword. After enter the specific keyword for remove outlier images. Finally remove the reduntant classes for next process. The visual similarities with the query image are selected from the images in the databases which are re-ranked automatically.

Consequently all the pictures in the pool are connected with the inquiry magic word as per the expression picture file document, they all have effectively processed semantic marks in the comparable semantic space indicated by the question watchword. 


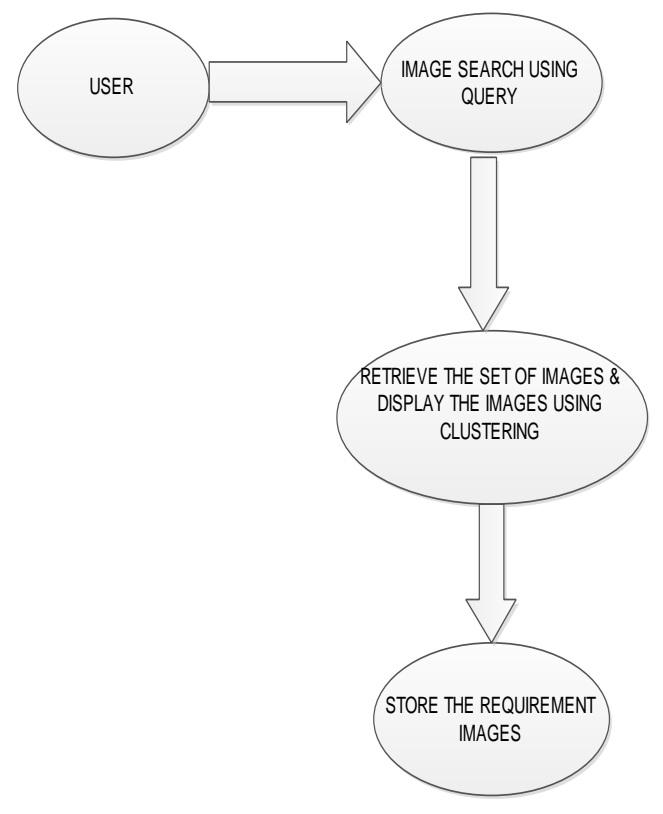

Fig.No.3 IMAGE RETRIEVAL

\section{G. Re-Ranking process of Image}

The user chooses a query image, these semantic signatures are used to compute similarities for image reranking. The Fig.No.4 Indicates semantic correlation of similar classes is being incorporated when computing the similarities. The proposed approach provides a natural way to integrate textual and visual features. Semantic signatures can also be determined from combined and textual features with those from optical features. Textual and visual features are in unique modalities. Without selecting images we can also apply query-specific semantic signature for image re-ranking. For this approach user need to give input as specific keyword. The query-specific semantic signatures significantly improve the image re-ranking by both accuracy and efficiency.

Method: kernel-based re-ranking, Information Bottleneck. H. Experimental Result

Fig.No.5 shows the pictures for testing the execution of re-positioning and the pictures of reference classes can be gathered at diverse time and from distinctive web search tools. Given an inquiry magic word, 1000 pictures are recovered from the entire web utilizing certain web crawler. We make three information sets to assess the execution of our methodology in diverse situations. In information set I, 120; 000 testing pictures for repositioning were gathered from the Bing Image Search utilizing 120 question pivotal words. These question catchphrases spread different subjects including creature, plant, nourishment, spot, individuals, occasion, object, scene, and so forth. The pictures of reference classes were additionally gathered from the Bing Image Search around the same time. Dataset II utilize the same testing pictures for re-positioning as in dataset I. Then again, its pictures of reference classes were gathered from the Google Image Search. In information set III, both testing pictures and pictures of reference classes were gathered from the Bing Image Search yet at diverse time (eleven months separated). All testing pictures for re-positioning are physically mark, while pictures of reference classes, whose number is much bigger, are not label.

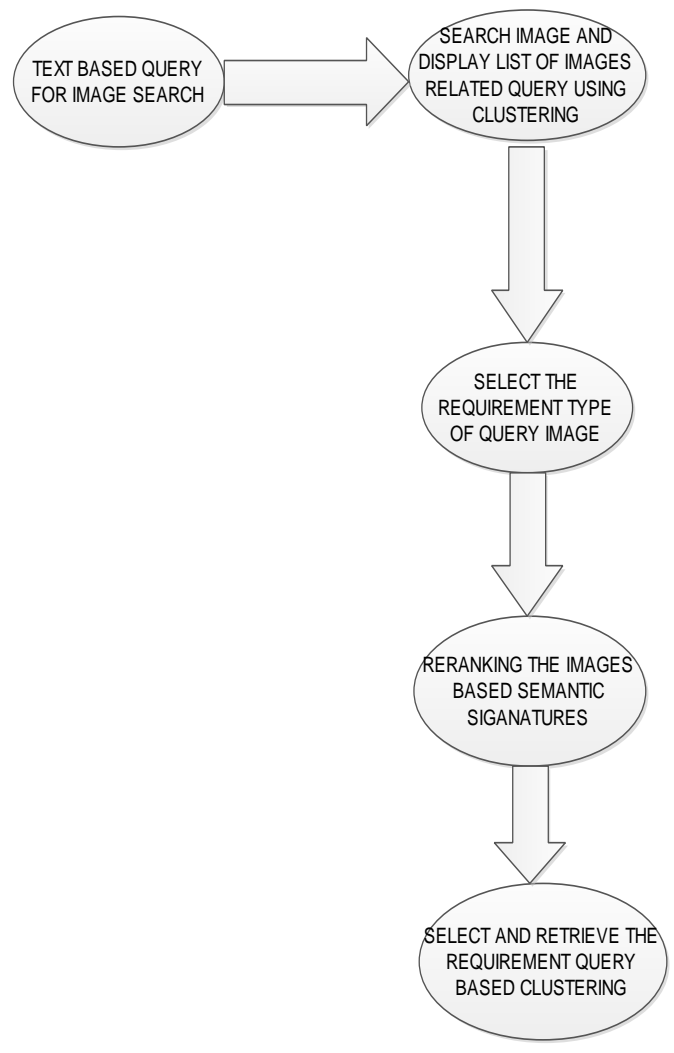

Fig.No.4 RE-RANKING PROCESS

\section{CONCLUSION AND FUTURE WORK}

A new re-ranking frame work is proposed to search images on the web where a user can give only one -click as feedback. Specific semantic signatures are used to combine visual similarities and features to get an adaptive query image. The visual and textual similarities are integrated and are combining for a better efficient image re-ranking. Re-ranking is done based on a single click on the image by the users. Hash codes are used to 
detect similar images and these images are eliminated by comparing their hash codes. For an improvised reranking of images specific query semantics spaces are used. The expansions of keywords are done by the projection of features into semantic space.

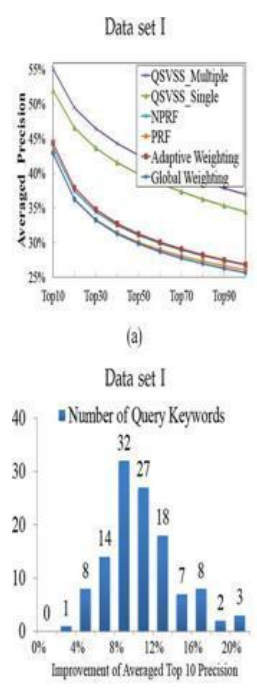

(d)

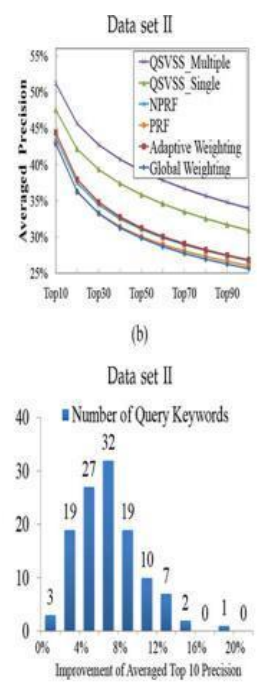

(e)

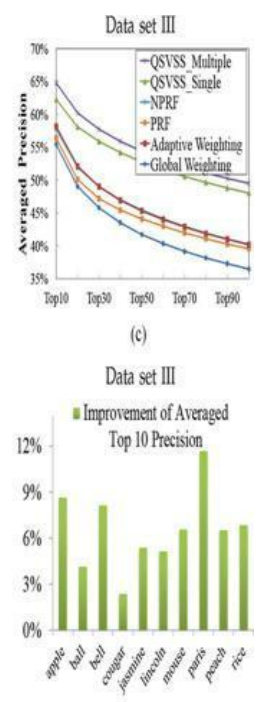

(f)
Fig.No. 5 Incorporating semantic correlations among reference classes. (a)-(c):Single visual semantic signatures with/without semantic correlation. visual \& textual semantic signatures with/without semantic correlation. (d)-(f):Multiple

User framework can be improved along several directions. To find the keyword expansions used to define reference classes can assimilate other log data and metadata besides the textual and visual features. For example, the co-occurrence information of keywords in user queries is useful and can be obtained in log data. In the future work identify the positive negative similarities and dimensions. In the future, How to re-ranking the online images for the efficiency and fast retrieval. In order to update the similar classes above time in an efficient way, how to acquire dynamic learning under our framework needs to be further investigated.

The future work, to achieve fast retrieval speed and make the retrieval system truly scalable to large image collections. We have try to Research some process, like good measurements of visual similarity, robust features, the importance of the user in the query process, and the gap between image understanding and image retrieval. . User use the image processing method for some advantages in the future work.Find the images in the easily way like the retrieve the particular images with image quality, history and details.

\section{REFERENCES}

[1] R. Datta, D. Jhoshi, and J.Z. Wang, "Image Retrieval: Ideas, Influences,and Trends of the New Age," ACM Computing Surveys,vol. 40, article 5, 2007.

[2] A.W.M. Smeulders, M. Woring, S. Santini, A. Gupta, and R. Jain,"Content-Based Image Retrieval," IEEE Trans. Pattern Analysis and Machine Intelligence, vol. 22, no. 12, pp. 1349-1380, Dec. 2000.

[3] X. Tang, K. Liu, J. Cuii, F. Wen, and X. Wang, "Intent Search: Capturing User Intention for One-Click Internet Image Search," IEEE Trans. Pattern Analysis and Machine Intelligence, vol. 34, no. 7, pp. 1342-1353, July 2012.

[4] Y. Kuo, W. Cheng, H. Lin, and W. Hsu, Unsupervised semantic feature discovery for image object retrieval and tag refinement, IEEE Trans on Multimedia, vol.14, pp.10791090, 2012.

[5] X. Wang, K. Liu, and X. Tang, Query-specific visual semantic spaces forweb image re-ranking, in Proc.CVPR, 2010.

[6] J. Cui, F. Wen, and X. Tang, Real time google and live image search re-ranking, in Proc. ACM Multimedia, pp.101-104, 2008.

[7] Bin Wang, Zhiwei Li, Mingjing Li, Wei-Ying Ma, LARGE SCALE DUPLICATE DETECTION FOR WEB IMAGESEARCH, IEEE, 1424403677/06/20.00,pp.110-116,2006.

[8] N. Dalal and B. Triggs, Histograms of oriented gradients for human detection, in Proc. IEEE Intl Conf.Computer Vision and Pattern Recognition,pp.204-207, 2005.

[9] A.Farhadi, I.Endres, and D.Hoiem, Attribute-centric recognition cross category generalization, in Proc. CVPR, pp.110-112,2010.

[10] N. Rasiwasia, P. J. Moreno, and N. Vasconcelos, Bridging the gap: Query by semantic example, IEEE Trans.onMultimedia,2007. 